\title{
Specific Effect of Polyunsaturated Fatty Acids on the Cholesterol-Poor Membrane Domain in a Model Membrane
}

\author{
Yoshinori Onuki, ${ }^{*}$ Chihiro Hagiwara, Ko Sugibayashi, and Kozo Takayama \\ Department of Pharmaceutics, Hoshi University; 2-4-41 Ebara, Shinagawa-ku, Tokyo 142-8501, Japan. \\ Received February 12, 2008; accepted May 28, 2008; published online May 30, 2008
}

\begin{abstract}
To understand more fully the effect of polyunsaturated fatty acids (PUFAs) on lipid bilayers, we investigated the effects of treatment with fatty acids on the properties of a model membrane. Three kinds of liposomes comprising dipalmitoylphosphatidylcholine (DPPC), dioleylphosphatidylcholine (DOPC), and cholesterol (Ch) were used as the model membrane, and the fluorescence anisotropy of 1,6-diphenyl-1,3,5-hexatriene (DPH) and detergent insolubility were determined. Characterization of the liposomes clarified that DPPC, DPPC/Ch, and DPPC/DOPC/Ch existed as solid-ordered phase $\left(L_{\beta}\right)$, liquid-ordered phase $\left(l_{0}\right)$, and a mixture of $l_{0}$ and liquiddisordered phase $\left(\mathrm{L}_{\alpha}\right)$ membranes at room temperature. Treatment with unsaturated fatty acids such as oleic acid (OA), eicosapentaenoic acid (EPA), and docosahexaenoic acid (DHA) markedly decreased the fluorescence anisotropy value and detergent insolubility. PUFAs and $O A$ had different effects on the model membranes. In DPPC liposomes, the most prominent change was induced by PUFAs, whereas, in DPPC/Ch and DPPC/DOPC/Ch liposomes, OA had a stronger effect than PUFAs. The effect of PUFAs was strongly affected by the amount of $\mathrm{Ch}$ in the membrane, which confirmed a specific effect of PUFAs on the Ch-poor membrane domain. We further explored the effect of fatty acids dispersed in a water-in-oil-in-water multiple emulsion and found that unsaturated fatty acids acted on the membranes even when incorporated in emulsion form. These findings suggest that treatment with PUFAs increases the segregation of ordered and disordered phase domains in membranes.
\end{abstract}

Key words polyunsaturated fatty acid; docosahexaenoic acid; eicosapentaenoic acid; cholesterol; liposome; water-in-oil-inwater multiple emulsion

Dietary intake of n-3 polyunsaturated fatty acids (PUFAs), such as docosahexaenoic acid (DHA, 22:6) and eicosapentaenoic acid (EPA, 20:5), is linked to the prevention of diseases such as cancer ${ }^{1)}$ and heart disease, ${ }^{2-4)}$ and PUFAs are needed for neurological and brain development. ${ }^{5)}$ The various action of PUFAs on biological functions have been paid attention. We have recently attempted to use these functional fatty acids as an absorption enhancer and have reported that intestinal absorption of insulin ${ }^{6-8)}$ and vancomycin ${ }^{9)}$ increase when administered with these fatty acids in a water-in-oil-inwater $(\mathrm{W} / \mathrm{O} / \mathrm{W})$ multiple emulsion. We have also reported that the enhancing effect of DHA and EPA is much stronger than that of other unsaturated fatty acids, such as oleic acid $(\mathrm{OA})$, and that they do not induce tissue damage. ${ }^{6-8)}$

When an emulsion is administered into the intestinal lumen, the PUFAs contained in the emulsion are taken up rapidly into the lipid bilayer of the intestinal mucosa, which changes the lipid packing. Although the mechanism remains unclear, alteration of the membrane properties is probably related to the strong enhancing effect. Model membranes are effective for investigating the details of membrane alterations. The composition of a model membrane can be manipulated fully to suit the purpose of the experiment, and the findings obtained are likely to be consistent. Previously, we investigated the effect of fatty acids on lipid bilayers using dipalmitoylphosphatidylcholine (DPPC) liposomes. ${ }^{10)}$ By detecting the phase transition temperature $\left(T_{\mathrm{m}}\right)$, fluorescence anisotropy, and detergent insolubility, we confirmed that unsaturated fatty acids such as PUFAs and OA are taken up in the membrane where they increase the membrane fluidity. Although the modifications of the bilayer physical properties were most pronounced with DHA and EPA treatment, considerable changes in the properties were also observed with
OA treatment. Thus, at present, we can not provide sufficient evidence to explain the mechanism of the strong enhancing effect of PUFAs. Further study is needed to understand more fully the effect of PUFAs on a membrane.

Recently, Shaikh et al. reported on the relationship between cholesterol $(\mathrm{Ch})$ and PUFAs in a model membrane and suggested an attractive hypothesis that PUFAs contained in the lipid bilayer are related to the formation of lipid microdomains such as lipid rafts. ${ }^{11-13)}$ They prepared liposomes comprising of DHA- or OA-containing phospholipids, sphingomyelin, and/or $\mathrm{Ch}$, and investigated various membrane properties of the model membranes using $2 \mathrm{H}-\mathrm{NMR}$, X-ray diffraction, and differential scanning calorimeter (DSC). In a series of experiments, Shaikh et al. confirmed that the miscibility of PUFAs with $\mathrm{Ch}$ is much lower than that of OA with Ch. They suggested that the low miscibility of membrane PUFAs with Ch stimulates the phase separation between the liquid-ordered and disordered domains, and that this may serve as a mechanism for triggering the formation of lipid microdomains such as lipid rafts. ${ }^{11)}$ The lipid raft is a membrane microdomain enriched in $\mathrm{Ch}$ and sphingolipid in the outer leaflet of the plasma membrane. Numerous signaling molecules such as GPI-anchored proteins ${ }^{14,15)}$ and receptor $^{16,17)}$ or nonreceptor-type tyrosine kinases ${ }^{16,18,19)}$ are located in lipid domains, and it is thought that these domains act as a platform for protein segregation and signal transduction in the plasma membrane. In addition, recent studies have shown that the lipid raft mediates intestinal absorption-related events such as P-glycoprotein-mediated efflux ${ }^{20)}$ and opening of tight junctions. ${ }^{21,22)}$ The lipid raft is assumed to exert a significant influence on intestinal drug absorption.

Considering the hypothesis of Shaikh et al., ${ }^{11)}$ one expects that application of PUFAs should also stimulate the lateral 
separation of distinct membranes. We investigated the effect of PUFAs on lipid bilayers using liposomes with different lipid composition. Three kinds of lipids are usually used as components of the membranes to imitate the plasma membrane. ${ }^{23,24)}$ These included lipids with high $T_{\mathrm{m}}$ (e.g., lipid with saturated acyl chains), lipids with low $T_{\mathrm{m}}$ (e.g., lipid with unsaturated acyl chains), and $\mathrm{Ch}$. We selected DPPC, dioleylphosphatidylcholine (DOPC), and $\mathrm{Ch}$ as the membrane components. We found that DPPC, a saturated lipid, formed an ordered phase membrane, whereas DOPC, an unsaturated lipid, formed a disordered phase membrane. Various fatty acids were applied to the liposomes, and the changes in the membrane properties were evaluated by measuring the membrane fluidity and detergent insolubility. We clarified the specific effect of PUFAs on the Ch-poor membrane domain. In addition, $\mathrm{W} / \mathrm{O} / \mathrm{W}$ multiple emulsions containing fatty acids were used as a model formulation, and the effects of fatty acids contained in the emulsion were investigated.

\section{Experimental}

Materials DPPC, DOPC, Ch, stearic acid (SA), polyoxyethylene(10)octylphenyl ether (Triton X-100), gelatin, triolein, sorbitan monooleate (Span 80), and DL- $\alpha$-tocopherol were purchased from Wako Pure Chemical Industries, Ltd. (Osaka, Japan). 1,6-Diphenyl-1,3,5-hexatriene (DPH) was purchased from Aldrich Chemical Co. (Milwaukee, WI, U.S.A.). OA and polyoxyethylene sorbitan monooleate (Tween 80) were purchased from Tokyo Kasei Kogyo Co., Ltd. (Tokyo, Japan). Egg yolk phospholipids (phosphatidylcholine and phosphatidylethanolamine) were purchased from Nippon Oil \& Fats Co., Ltd. (Tokyo, Japan). DHA (purity 99.0\%) and EPA (purity 99.0\%) were provided by Nippon Suisan Kaisya, Ltd. (Tokyo, Japan). All other chemicals were of analytical grade and commercially available.

Preparation of Liposome DPPC, DPPC/Ch and DPPC/DOPC/Ch liposomes were prepared as reported previously. ${ }^{10)}$ The molar ratios of lipids of DPPC/Ch and DPPC/DOPC/Ch liposomes were $0.7: 0.3$ and $0.35: 0.35$ : 0.3 , respectively. In brief, the designated amounts of lipids dissolved in chloroform were pipetted into a flask, and the chloroform was removed by evaporation at room temperature under a nitrogen stream. This procedure resulted in the formation of a thin lipid film on the inside wall of the flask. The film was stored overnight in a vacuum desiccator to ensure the complete evaporation of chloroform. Ten milliliters of phosphate-buffered saline (PBS, $\mathrm{pH}$ 7.4) was added to the flask and the lipids were hydrated for $30 \mathrm{~min}$. The suspension was sonicated for $10 \mathrm{~min}$ at about $60^{\circ} \mathrm{C}$ using a bath-type sonicator. The liposome suspension was prepared at a total lipid concentration of $10 \mathrm{~mm}$ and stored at room temperature until used in the experiments.

Detergent-Resistance Studies Fatty acids were applied to liposomes in the form of suspensions and W/O/W multiple emulsions. Fatty acid suspensions were prepared by suspending the designated amounts of fatty acids such as SA, OA, EPA, and DHA in a mixture of methanol and PBS $(1: 1$, $\mathrm{v} / \mathrm{v})$. W/O/W multiple emulsions were prepared by a two-step emulsification procedure using a homogenizer (Ace Homogenizer, Nihonseiki Kaisha, Tokyo, Japan) according to the method reported previously. ${ }^{25)}$ Briefly, purified water containing $5 \%$ gelatin and $3 \%$ Tween 80 were used for the inner and outer aqueous phases, respectively. The oily phase comprised $0.06 \%$ DL- $\alpha$-tocopherol, $5 \%$ egg yolk phospholipids (phosphatidylcholine:phosphatidylethanolamine, $7: 3$ ), 10\% fatty acid, and 20\% Span 80 . An appropriate amount of triolein was added to adjust the weight of the oily phase. The weight ratio of each phase was inner aqueous phase : oily phase : outer aqueous phase, $1: 4: 15$. Each emulsion was prepared fresh just before the experiment. Fatty acids were applied to the liposomes as described previously. ${ }^{26)}$ Two hundred and fifty microliters of fatty acid suspension or emulsion was added to $1000 \mu \mathrm{l}$ of liposome suspensions, and this mixture was incubated for $2 \mathrm{~h}$ at $37^{\circ} \mathrm{C}$.

Detergent resistant study was conducted according to the method reported by Wang et al. ${ }^{27)}$ After application of fatty acids, $750 \mu 1$ of $10 \%$ Triton X100 solution was added to $1250 \mu \mathrm{l}$ of a liposome suspension treated with fatty acid, and the sample was incubated at $25^{\circ} \mathrm{C}$ for $2 \mathrm{~h}$. The sample was centrifuged at $13400 \times \boldsymbol{g}$ for $2 \mathrm{~min}$ to separate the supernatant and pellet. The supernatant was removed, and the pellet was resuspended in an equal volume of fresh PBS. The sample was diluted 100 times with PBS, and the optical density (OD) at $400 \mathrm{~nm}$ was measured using a spectrophotometer (Ubest 30, JASCO, Tokyo, Japan). The OD at $400 \mathrm{~nm}$ of a freshly prepared liposome suspension was also measured, and this value was used as the initial level. Detergent insolubility was calculated as the OD after the addition of Triton X-100 divided by the initial level.

Fluorescence Anisotropy Measurement The liposome was labeled with DPH by adding $10 \mu \mathrm{l}$ of $10 \mathrm{~mm}$ freshly prepared DPH stock solution in tetrahydrofuran to $1000 \mu \mathrm{l}$ of liposome suspension and then incubating at $37^{\circ} \mathrm{C}$ for $2 \mathrm{~h}$ in the dark to complete the labeling. The fluorescence anisotropy of DPH in the liposomes was measured with a fluorescence spectrophotometer (Hitachi F-450, Hitachi Co., Ltd., Tokyo, Japan) at an excitation wavelength of $351 \mathrm{~nm}$ and an emission wavelength of $430 \mathrm{~nm}$. The absorbance of all samples at $400 \mathrm{~nm}$ was set to $<0.50$ using PBS as the dilution medium to measure the fluorescence. The steady-state fluorescent anisotropy was calculated using the following equation:

$$
r=\frac{I_{\mathrm{VV}}-I_{\mathrm{VH}}}{I_{\mathrm{VV}}+2 I_{\mathrm{VH}}}
$$

where $r$ is anisotropy, and $I_{\mathrm{VV}}$ and $I_{\mathrm{VH}}$ are the intensity measured in directions parallel and perpendicular to the polarized exciting light, respectively.

Fluorescence anisotropy was also measured of fatty acid treated liposomes and detergent resistant membranes (DRMs). The application of fatty acids to DPH-labeled liposomes and the separation of DRMs from liposomes were performed according to the methods described above. Liposomes and DRMs were washed with PBS and centrifuged at $13000 \mathrm{rpm}$ for 2 min, and the obtained pellets were resuspended in PBS for measurement.

\section{Results}

Membrane Fluidity of Liposomes and DRMs Figure 1 shows the fluorescence anisotropy of DPH in liposomes as a function of temperature. The value of the DPPC liposome decreased markedly at about $44{ }^{\circ} \mathrm{C}$ from 0.35 to less than 0.20 , and a phase transition of DPPC was observed. In contrast, the values of $\mathrm{DPPC} / \mathrm{Ch}$ and $\mathrm{DPPC} / \mathrm{DOPC} / \mathrm{Ch}$ liposomes changed little with changes in temperature. The fluorescence anisotropy values were much higher in the $\mathrm{DPPC} / \mathrm{Ch}$ liposomes than in $\mathrm{DPPC} / \mathrm{DOPC} / \mathrm{Ch}$ liposomes over the experimental period.

We next separated DRMs from liposomes by incubating with Triton X-100 and measured their fluorescence anisotropy values at $37^{\circ} \mathrm{C}$ (Fig. 2). The fluorescence anisotropy values of the DRMs separated from DPPC and $\mathrm{DPPC} / \mathrm{Ch}$ liposomes were $0.377 \pm 0.009$ and $0.347 \pm 0.011$ respectively. These values did not differ from those of the original liposomes. The value was much higher in DRMs

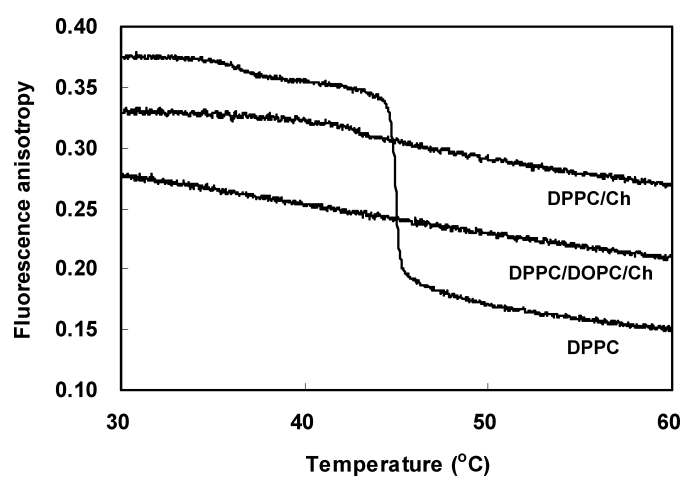

Fig. 1. Fluorescence Anisotropy of DPH in the DPPC, DPPC/Ch, and DPPC/DOPC/Ch Liposomes

Lipid compositions of DPPC/Ch and DPPC/DOPC/Ch liposomes were $0.7: 0.3$ and $0.35: 0.35: 0.3$, respectively. The temperature was scanned at $1{ }^{\circ} \mathrm{C} / \mathrm{min}$. 
separated from DPPC/DOPC/Ch liposomes $(0.345 \pm 0.004)$ than from the original liposomes $(0.229 \pm 0.009)$ and was similar to those of DRMs from DPPC and DPPC/Ch liposomes.

Effect of Fatty Acids on Distinct Membranes We investigated the effect of fatty acids on distinct membranes. In a previous study using DPPC liposomes, we reported that unsaturated fatty acids increase the membrane fluidity in a dose-dependent manner. ${ }^{10)}$ Based on our earlier study, we fixed the treatment amount of fatty acids at $30 \mathrm{~mol} \%$ relative to the amount of lipid in the liposomes. A mixture of methanol and PBS without any fatty acid was applied to the liposomes as a control.

Figure 3 and Table 1 show the effect of fatty acids on the



Fig. 2. Fluorescence Anisotropy of DPH in the Original Liposomes ( $\square$ ) or Detergent Resistant Membranes (匹) at $37^{\circ} \mathrm{C}$

The lipid compositions of DPPC/Ch and DPPC/DOPC/Ch liposomes were $0.7: 0.3$ and $0.35: 0.35: 0.3$, respectively. Detergent-resistant membranes were obtained by applying TX-100 to liposomes for $2 \mathrm{~h}$ at $25^{\circ} \mathrm{C}$. Each value represents the mean \pm S.D. $(n=3)$. $* * p<0.01$. fluorescence anisotropy of DPH. Treatment with unsaturated fatty acids such as OA, EPA, or DHA decreased the fluorescence anisotropy values of all membranes. DPPC liposomes were the most sensitive to the fluidizing effect of unsaturated fatty acids. PUFAs had much greater impact on the DPPC liposome than did OA. The saturated $\mathrm{C} 18$ fatty acid SA had no effect on any membrane.

Figure 4 and Table 2 show the detergent insolubility of fatty acid-treated liposomes. The control values of DPPC,

Table 1. Fluorescence Anisotropy of DPH in Fatty Acid-Treated Liposomes at $37^{\circ} \mathrm{C}$

\begin{tabular}{lll}
\hline \hline Lipid composition & Fatty acids & Fluorescence anisotropy \\
\hline \multirow{2}{*}{ DPPC } & Control & $0.357 \pm 0.015$ \\
& SA & $0.365 \pm 0.005$ \\
OA & $0.293 \pm 0.008^{*, * *}$ \\
& EPA & $0.234 \pm 0.005^{* * * * * * *}$ \\
& DHA & $0.223 \pm 0.003^{*, * *, * * *}$ \\
DPPC/Ch & Control & $0.350 \pm 0.005$ \\
& SA & $0.332 \pm 0.003$ \\
& OA & $0.315 \pm 0.003 *, * *$ \\
DPPC/DOPC/Ch & EPA & $0.323 \pm 0.002^{*}$ \\
& DHA & $0.324 \pm 0.002^{*}$ \\
& Control & $0.229 \pm 0.004$ \\
& SA & $0.224 \pm 0.002$ \\
& OA & $0.200 \pm 0.003^{*} * *$ \\
& EPA & $0.197 \pm 0.003^{*} * *$ \\
& DHA & $0.201 \pm 0.020$
\end{tabular}

Thirty mole percent of fatty acid relative to the total lipids of the liposomes was applied to the liposomes in the form of a suspension for $2 \mathrm{~h}$ at $37^{\circ} \mathrm{C}$. Each value represents the mean \pm S.D. $(n=3)$. $* p<0.01 v s$. control; $* * p<0.01$ vs. stearic acid; $* * * p<0.01 v s$. oleic acid. (a)



(b)



(c)

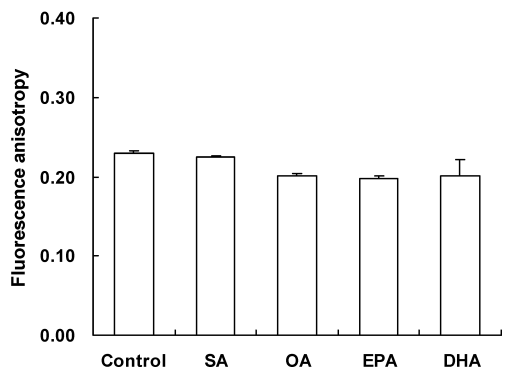

Fig. 3. Effect of Fatty Acids on the Fluorescence Anisotropy of DPH in Liposomes at $37^{\circ} \mathrm{C}$

Thirty mole percent of fatty acid relative to total lipids of liposome was applied to the liposomes in the form of a suspension. (a) DPPC, (b) DPPC/Ch, and (c) DPPC/DOPC/Ch. Each value represents the mean \pm S.D. $(n=3)$.

(a)

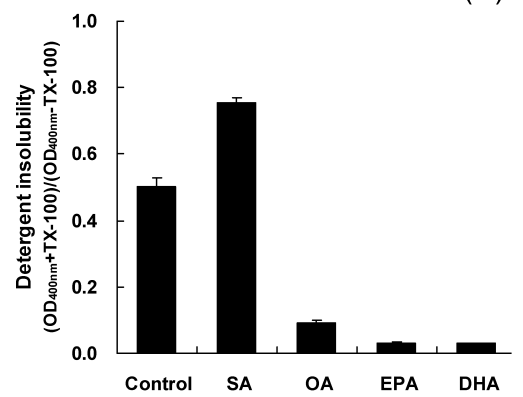

\section{)}

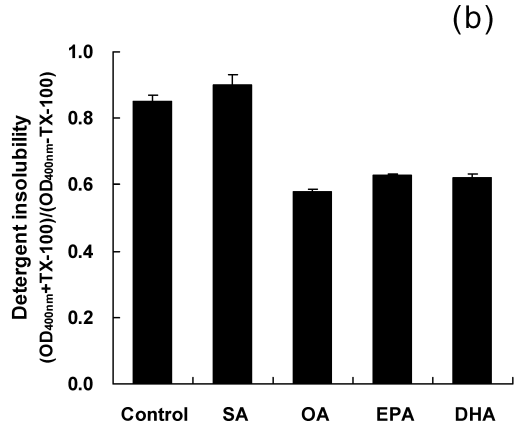

(b)

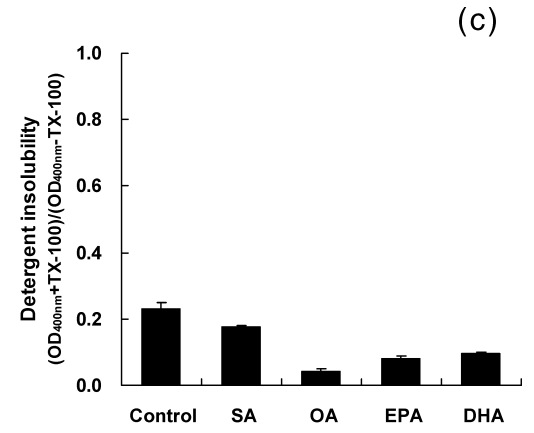

Fig. 4. Effect of Fatty Acids on Detergent Insolubility

Thirty mole percent of fatty acid relative to total lipids of the liposomes was applied to the liposomes in the form of a suspension. (a) DPPC, (b) DPPC/Ch, and (c) DPPC/DOPC/Ch. Each value represents the mean \pm S.D. $(n=3)$. 
$\mathrm{DPPC} / \mathrm{Ch}$, and DPPC/DOPC/Ch liposomes were $0.501 \pm$ $0.027,0.851 \pm 0.019$ and $0.229 \pm 0.021$, respectively. A large amount of lipid in the DPPC/DOPC/Ch liposomes was dissolved in the Triton X-100, whereas the DPPC/Ch liposomes were stable to solubilization by Triton X-100. The values decreased after treatment with unsaturated fatty acids. In DPPC liposomes, the lowest amounts of DRMs were obtained from liposomes treated with PUFAs such as EPA and DHA (Fig. $4 \mathrm{a}$, Table 2). In contrast, in DPPC/Ch and DPPC/DOPC/Ch liposomes, the amount of DRM was lower in OA-treated liposomes than in PUFA-treated liposomes (Figs. 4b, c, Table 2). These data show that the actions of PUFAs and OA differed. In the SA-treated liposomes, the DRM amount tended to be higher compared with the controls (Table 2).

Effect of Fatty Acids Contained in W/O/W Emulsion on Distinct Membranes Figure 5 and Table 3 show the fluorescence anisotropy of DPH after treatment with a $\mathrm{W} / \mathrm{O} / \mathrm{W}$ emulsion containing various fatty acids. Fatty acidfree emulsion treatment was used as a control. The control values of DPPC, DPPC/Ch, and DPPC/DOPC/Ch liposomes were $0.305 \pm 0.007,0.290 \pm 0.005$ and $0.199 \pm 0.011$, respectively (Table 3 ). These values were much lower than the control levels in the form of suspension shown in Table 1. In

Table 2. Detergent Insolubility Observed from Liposomes Treated with Various Fatty Acids

\begin{tabular}{|c|c|c|}
\hline Lipid composition & Fatty acids & $\begin{array}{l}\text { Detergent insolubility } \\
\left(\mathrm{OD}_{400 \mathrm{~nm}}+\mathrm{TX}-100\right) \\
/\left(\mathrm{OD}_{400 \mathrm{~nm}}-\mathrm{TX}-100\right)\end{array}$ \\
\hline \multirow[t]{5}{*}{ DPPC } & Control & $0.501 \pm 0.027$ \\
\hline & SA & $0.755 \pm 0.014 *$ \\
\hline & $\mathrm{OA}$ & $0.092 \pm 0.006^{*, * *}$ \\
\hline & EPA & $0.030 \pm 0.002 *, * *, * * *$ \\
\hline & DHA & $0.030 \pm 0.004 *, * *, * * *$ \\
\hline \multirow[t]{5}{*}{$\mathrm{DPPC} / \mathrm{Ch}$} & Control & $0.851 \pm 0.019$ \\
\hline & $\mathrm{SA}$ & $0.900 \pm 0.029$ \\
\hline & $\mathrm{OA}$ & $0.577 \pm 0.009 * * *$ \\
\hline & EPA & $0.619 \pm 0.014^{*, * *, * * *}$ \\
\hline & DHA & $0.628 \pm 0.005^{* * * *}$ \\
\hline \multirow[t]{5}{*}{ DPPC/DOPC/Ch } & Control & $0.229 \pm 0.021$ \\
\hline & SA & $0.177 \pm 0.003$ \\
\hline & $\mathrm{OA}$ & $0.044 \pm 0.006^{*, * *}$ \\
\hline & EPA & $0.095 \pm 0.003 *, * *, * * *$ \\
\hline & DHA & $0.079 \pm 0.010^{*, * *, * * *}$ \\
\hline
\end{tabular}

Thirty mole percent of fatty acid relative to total lipids of liposome was applied to the liposomes in the form of a suspension for $2 \mathrm{~h}$ at $37^{\circ} \mathrm{C}$. Each value represents the mean \pm S.D. $(n=3)$. $* p<0.01$, vs. control; $* * p<0.01$, vs. stearic acid; $* * * p<0.01$, vs. oleic acid.
DPPC liposomes, the fluorescence anisotropy values decreased further after treatment with unsaturated fatty acidcontaining emulsions. In contrast, in DPPC/Ch and DPPC/ DOPC/Ch liposomes, application of unsaturated fatty acids hardly influenced the fluorescence anisotropy values (Table $3)$.

Figure 6 and Table 4 show the detergent insolubility of emulsion-treated liposomes. As the same as the fluorescence anisotropy measurement, the emulsion affected liposomes more strongly than did the fatty acid suspension (Table 2), and lower values were generally observed. The DPPC liposome was an especially sensitive membrane. Even when fatty acid-free and SA emulsion were applied, the obtained values were very low $(0.045 \pm 0.002$ or $0.101 \pm 0.030)$. Treatment with unsaturated fatty acid-containing emulsion progressively decreased the detergent insolubility. The effect of unsaturated fatty acids contained in the emulsion was similar to that of unsaturated fatty acids dispersed in suspension. In DPPC liposomes, the lowest amount was obtained from PUFA emulsion-treated liposomes. In contrast, in the $\mathrm{DPPC} / \mathrm{Ch}$ and DPPC/DOPC/Ch liposomes, the lowest DRM amount was obtained from OA-emulsion treated liposomes (Table 4).

Table 3. Fluorescence Anisotropy of DPH in Liposomes after Treatment with $\mathrm{W} / \mathrm{O} / \mathrm{W}$ Emulsions Containing Various Fatty Acids at $37^{\circ} \mathrm{C}$

\begin{tabular}{lll}
\hline \hline Lipid composition & Fatty acids & Fluorescence anisotropy \\
\hline \multirow{2}{*}{ DPPC } & Control & $0.305 \pm 0.007$ \\
& SA & $0.346 \pm 0.002^{*}$ \\
OA & $0.278 \pm 0.005^{*, * *}$ \\
& EPA & $0.208 \pm 0.006^{*, * * * * *}$ \\
& DHA & $0.210 \pm 0.005^{*, * * * * * *}$ \\
DPPC/Ch & Control & $0.290 \pm 0.005$ \\
& SA & $0.286 \pm 0.004$ \\
& OA & $0.265 \pm 0.003^{*, * *}$ \\
& EPA & $0.315 \pm 0.014^{* * *}$ \\
DPPC/DOPC/Ch & DHA & $0.302 \pm 0.014^{*}$ \\
& Control & $0.199 \pm 0.011$ \\
& SA & $0.203 \pm 0.004$ \\
& OA & $0.173 \pm 0.016$ \\
& EPA & $0.179 \pm 0.005^{* *}$ \\
& DHA & $0.198 \pm 0.022$
\end{tabular}

Fatty acids were applied to the liposomes in the form of a W/O/W emulsion for $2 \mathrm{~h}$ at $37^{\circ} \mathrm{C}$. Each value represents the mean \pm S.D. $(n=3) . * p<0.01 v$ s. control; $* * p<0.01$ vs. stearic acid; $* * * p<0.01$ vs. oleic acid. (a)

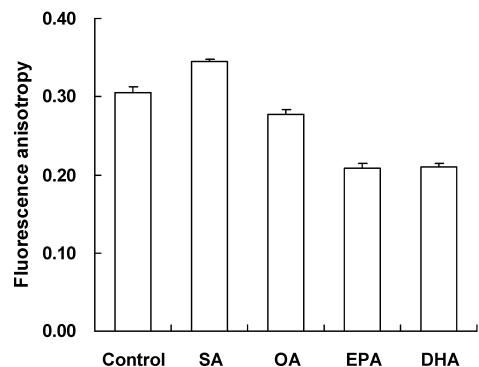

(b)

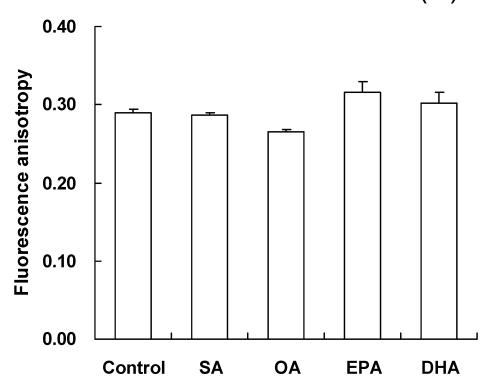

(c)

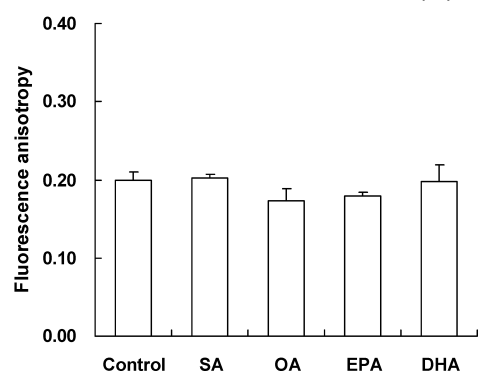

Fig. 5. Effect of Fatty Acids Contained in W/O/W Emulsions on the Fluorescence Anisotropy of DPH in Liposomes at $37^{\circ} \mathrm{C}$

Fatty acids were applied to the liposomes in the form of a W/O/W emulsion. (a) DPPC, (b) DPPC/Ch, and (c) DPPC/DOPC/Ch. Each value represents the mean \pm S.D. ( $n=3$ ). 
(a)



(b)

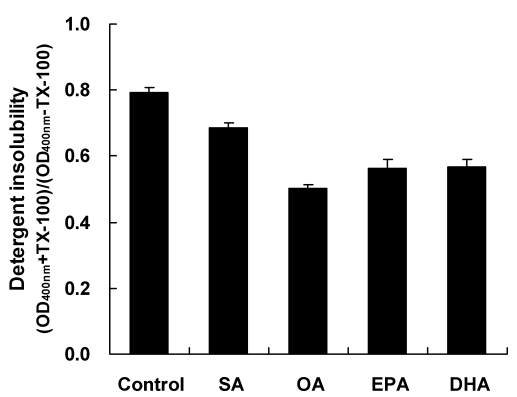

(c)

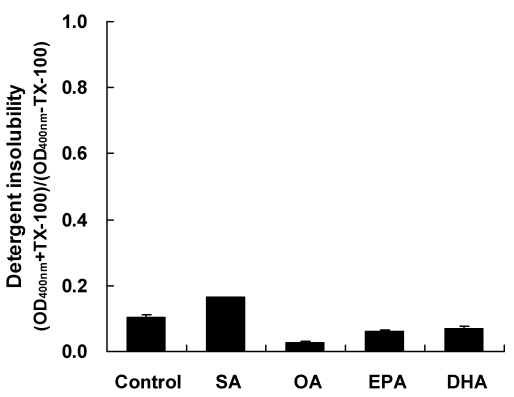

Fig. 6. Effect of Fatty Acids Contained in W/O/W Emulsions on Detergent Insolubility

Fatty acids were applied to the liposomes in the form of a W/O/W emulsion. (a) DPPC, (b) DPPC/Ch, and (c) DPPC/DOPC/Ch. Each value represents the mean \pm S.D. ( $n=3$ ).

Table 4. Fluorescence Anisotropy of DPH in Liposomes after Treatment with W/O/W Emulsions Containing Various Fatty Acids

\begin{tabular}{lll}
\hline \hline Lipid composition & Fatty acids & $\begin{array}{c}\text { Detergent insolubility } \\
\left(\mathrm{OD}_{400 \mathrm{~nm}}+\mathrm{TX}-100\right) \\
/\left(\mathrm{OD}_{400 \mathrm{~nm}}-\mathrm{TX}-100\right)\end{array}$ \\
\hline DPPC & Control & $0.045 \pm 0.002$ \\
& SA & $0.101 \pm 0.030$ \\
& OA & $0.012 \pm 0.005^{*}$ \\
& EPA & $0.007 \pm 0.003^{*}$ \\
& DHA & $0.008 \pm 0.008^{*, * *}$ \\
DPPC/Ch & Control & $0.794 \pm 0.014$ \\
& SA & $0.688 \pm 0.015^{*}$ \\
& OA & $0.502 \pm 0.011^{*, * *}$ \\
& EPA & $0.564 \pm 0.026^{*, * *}$ \\
& DHA & $0.568 \pm 0.020^{*, * *, * * *}$ \\
& Control & $0.105 \pm 0.007$ \\
& SA & $0.163 \pm 0.000^{*}$ \\
& OA & $0.027 \pm 0.003^{*, * *}$ \\
& EPA & $0.063 \pm 0.003^{*, * * * * *}$ \\
& DHA & $0.070 \pm 0.006^{*, * *, * * *}$ \\
\end{tabular}

Fatty acids were applied to the liposomes in the form of a $\mathrm{W} / \mathrm{O} / \mathrm{W}$ emulsion for $2 \mathrm{~h}$ at $37^{\circ} \mathrm{C}$. Each value represents the mean \pm S.D. $(n=3) . * p<0.01 v s$. control; $* * p<0.01$ $v s$. stearic acid; $* * * p<0.01 v s$. oleic acid.

\section{Discussion}

Lipid bilayers are generally classified into three different phases in order of increasing fluidity: a solid-ordered phase $\left(\mathrm{L}_{\beta}\right)$, a liquid-ordered phase $\left(\mathrm{l}_{\mathrm{o}}\right)$, and a liquid-disordered phase $\left(\mathrm{L}_{\alpha}\right){ }^{28)} \mathrm{L}_{\beta}$ and $\mathrm{L}_{\alpha}$ phases are also called gel and liquid crystalline phases, respectively. When temperature rises, the Ch-poor membrane changes from an $\mathrm{L}_{\beta}$ phase to $\mathrm{L}_{\alpha}$ phase at $T_{\mathrm{m}}$. Ordered and tight packing are characteristics of the $\mathrm{L}_{\beta}$ phase membrane, whereas fast axial rotation and high lateral mobility are observed in the $\mathrm{L}_{\alpha}$ phase membrane. The Chrich membrane exists as an $1_{o}$ phase membrane; this phase is intermediate between the $\mathrm{L}_{\alpha}$ and $\mathrm{L}_{\beta}$ phases. The ordered packing show similar to the $\mathrm{L}_{\beta}$ phase, but fast axial rotation and high lateral mobility are more akin to the $\mathrm{L}_{\alpha}$ state. The lipid raft is assumed to exist as an $1_{0}$ phase membrane in the plasma membrane.

We used DPPC, DPPC/Ch, and DPPC/DOPC/Ch liposomes as model membranes. The DPPC liposome is known to exist as an $\mathrm{L}_{\beta}$ phase membrane below $T_{\mathrm{m}}$. The DPPC liposome changes from an $\mathrm{L}_{\beta}$ phase membrane to an $1_{\mathrm{o}}$ phase membrane by incorporating $\mathrm{Ch}$ into the membrane. In a previous study, the intensity of the endothermic peak representing the phase transition of DPPC decreased with increasing
Ch concentration, and the peak disappeared completely when the $\mathrm{Ch}$ concentration reached $30 \mathrm{~mol} \%$ relative to the total amount of lipid. ${ }^{29)}$ The DPPC/Ch liposomes used in this study contained a lipid molar ratio of $0.7: 0.3$, and the entire membrane was expected to exist as an $1_{0}$ phase membrane. The DPPC/DOPC/Ch liposome was designed as a mixture of $1_{\mathrm{o}}$ and $\mathrm{L}_{\alpha}$ phase domains. DOPC is a low $-T_{\mathrm{m}}$ lipid by nature and forms a disordered phase domains. Recent studies show that several lipid phase domains coexist in even model membranes. $^{30-33)}$

To confirm the characteristics of the liposomes, we first evaluated their fluorescence anisotropy. As shown in Fig. 1, the fluorescence anisotropy decreased markedly in the DPPC liposomes at $44^{\circ} \mathrm{C}$. This represents the phase transition of DPPC from the $\mathrm{L}_{\beta}$ phase to the $\mathrm{L}_{\alpha}$ phase. The DPPC liposome no doubt exists as an $\mathrm{L}_{\beta}$ phase membrane below the $T_{\mathrm{m}}$. In the $\mathrm{DPPC} / \mathrm{Ch}$ and $\mathrm{DPPC} / \mathrm{DOPC} / \mathrm{Ch}$ liposomes, no marked decrease in fluorescence anisotropy value was observed, implying that the DPPC-rich domain in these liposomes was changed completely to the $1_{\mathrm{o}}$ phase membrane. In addition, the fluorescence anisotropy values were much lower in the DPPC/DOPC/Ch liposomes than in the DPPC/Ch liposomes, probably because of enrichment of the disordered phase domain in DOPC in the liposomes. We also measured the fluorescence anisotropy in DRMs separated from liposomes. The detergent insolubility study was based on the observation that ordered phase domains tend to resist solubilization by nonionic detergents such as Triton X-100, whereas disordered phase domains dissolve in these detergents. The insoluble membrane fraction, DRM, can be regarded as comprising tightly packed domains that were present in the sample before the addition of detergent. London et al. used detergent insolubility as an index of the formation of the ordered phase domain (lipid raft) in a model membrane. ${ }^{27,34)}$ As shown in Fig. 2, the fluorescence anisotropy values of every DRM were equal to the values of the ordered phase membranes such as DPPC and DPPC/Ch liposomes, from which we obtained a tight packing membrane fraction as a DRM. Interestingly, the values for DRMs separated from DPPC/DOPC/Ch liposomes increased drastically and came close to those obtained from DPPC and DPPC/Ch liposomes (Fig. 2). This indicates the coexistence of the ordered and disordered phase domains in DPPC/DOPC/Ch liposomes. The disordered phase domain was dissolved in Triton X-100, leaving the ordered phase domain. Thus, we obtained the distinct membranes that we had anticipated. 
We also evaluated the effect of fatty acids on liposomes. Since the effect of unsaturated fatty acids on DPPC liposome has already been investigated in the previous article, ${ }^{10}$ this study focused on the effect of fatty acids on different phase membranes. SA treatment tended to increase the values of fluorescence anisotropy of the DPH and DRM amounts (Figs. 3, 4). Because SA is a saturated fatty acid composed of a longer hydrocarbon chain than DPPC, the packing of the membranes might be tighter after treatment with SA. Unsaturated fatty acid significantly affected the membrane properties in a complicated manner. In DPPC liposomes, treatment with unsaturated fatty acids markedly decreased the fluorescence anisotropy value and detergent insolubility (Figs. 3, 4). PUFAs such as DHA and EPA had a strong effect on the membrane. The packing of DPPC liposome is very tight. Once applied to the DPPC liposome, unsaturated fatty acids are taken up into the lipid bilayer, reducing the van der Waals interactions between the phospholipid hydrocarbon chains because of their kinked structure, ${ }^{35)}$ which results in an increased membrane fluidity and reducing packing. We speculate that two mechanisms may be responsible as the main cause of the strongest effect of PUFAs. One is related to the difference in the molecular structure. The structure of PUFAs is bulkier than that of OA because of polyunsaturation, which should fluidize the membrane packing more strongly. The other mechanism relates to the greater incorporation of PUFAs into the lipid bilayer compared with OA. We previously confirmed that the incorporated amount of fatty acids into the membrane was similar in these membranes. ${ }^{10}$ Thus, the stronger effect of PUFAs probably arises from the molecular structure, such as the kinked conformation contributed by the double bond. Stillwell et al. reported that unsaturated fatty acid as a component of a membrane increases the membrane fluidity and strengthens the fluidizing effect with an increasing degree of unsaturation. ${ }^{35,36)}$ Our result is consistent with these findings.

In contrast to the situation in DPPC liposomes, we observed different actions of unsaturated fatty acids in DPPC/Ch and DPPC/DOPC/Ch liposomes. First, the fluidizing effect of unsaturated fatty acids became weaker. The interaction between unsaturated fatty acids and the Ch-rich domain probably became difficult because their properties are too different from each other. The influence of unsaturated fatty acids on detergent insolubility changed and the lowest DRM amount was obtained from OA-treated liposomes (Figs. 4b, c). Shaikh et al. confirmed that the affinity of DHA-containing phospholipids for $\mathrm{Ch}$ is much lower than in OA-containing phospholipids. ${ }^{11,12)}$ Although it is still remains unclear, they suggested that this mechanism acts as a driving force to segregate ordered and disordered membrane domains in the membrane surface, triggering the formation of lipid rafts. ${ }^{11,12)}$ Our findings show a relationship to the amount of $\mathrm{Ch}$ contained in the membrane. That is, when the membrane was $\mathrm{Ch}$ poor (e.g., $\mathrm{L}_{\alpha}$ and $\mathrm{L}_{\beta}$ phase membrane), both PUFAs and OA interacted easily with the membrane, and considerable amounts of fatty acid were taken up. At the time, PUFAs affect on the membrane much stronger than OA because of the kinked conformation contributed by the double bond. In contrast, when the membrane was rich in $\mathrm{Ch}$ (e.g., $1_{\mathrm{o}}$ phase membrane), although OA was taken up to a certain degree, PUFAs did not easily approach the Ch-rich membrane. According to this mechanism, PUFAs act on Chpoor membranes with high selectivity.

We also evaluated the effect of fatty acid incorporated in an emulsion, because strong enhancing effect of PUFAs on intestinal drug absorption were observed using the dosage form. We found that the effect of unsaturated fatty acid incorporated in the emulsion gave similar results as those when fatty acids were applied in the form of a suspension (Fig. 6). This confirmed that unsaturated fatty acids act efficiently on the membrane even when included in emulsion form. The control emulsion (fatty acid-free emulsion) also markedly modified the lipid bilayer structure (Tables 3,4). This probably reflects the unsaturated lipids contained in the emulsion, such as triolein and egg yolk phospholipids.

Our experiments confirmed the specific effect of PUFAs on Ch-poor membranes. According to the hypothesis of Shaikh et al., ${ }^{11)}$ applied PUFAs may also enhance lipid raft formation. Several recent reports show the involvement of PUFAs in the formation and function of lipid rafts in cultured cells or animals. For instance, Duraisamy et al. applied $100 \mu \mathrm{M}$ of DHA to Caco-2 cell monolayer for $\left.8 \mathrm{~d} .{ }^{37}\right)$ The amount of PUFAs in the plasma membrane increased markedly, and the DHA content of the separated lipid raft component increased 25-fold. In addition, lipid raft associated proteins such as Src and Fyn were excluded from lipid raft. Li et al. cultured $\mathrm{T}$ cells in medium with addition of $50 \mu \mathrm{M}$ of DHA for $2 \mathrm{~d}$ and then investigated lipid raft composition and localization of interleukin-2 (IL-2) receptor signaling molecules. $\left.{ }^{38}\right)$ Treatment with DHA increased the amount of PUFAs in the lipid raft, and some membrane proteins such as the IL-2 receptor or IL-2-induced Janus kinase-signal transducer and activator of transcription (STAT) were displaced from the lipid raft fractions to the soluble membrane fractions (disordered phase domain). Recently, they reported that EPA also changed lipid composition of membrane lipid raft and $\mathrm{T}$ cell function. ${ }^{39)}$ Schley et al. conducted experiments using MDA-MB-231 human breast cancer cells, and observed similar evidence like increase in the PUFAs amount in the lipid raft fraction and change in the lipid composition. ${ }^{40)}$ Fan et al. investigated the influence of dietary intake of n-3 PUFAs on $\mathrm{T}$ cell microdomain lipid composition in mice. After feeding $4 \mathrm{~g}$ or $100 \mathrm{~g}$ of fish oil to mice for $14 \mathrm{~d}$, the n-3 PUFA content in splenic T cell lipid rafts increased, and the sphingomyelin content decreased. ${ }^{41,42)}$ The increase in the amount of PUFAs in lipid rafts and displacement of the lipid raft-associated proteins imply that the application of PUFAs disturbed the lipid rafts in the plasma membrane. To date, no positive evidence has been reported to show that PUFAs enhance lipid raft formation. We assume that these results are strongly associated with the fluidizing effect of PUFAs on the Ch-poor membrane. The plasma membrane is a complicated system, and it is difficult to perceive slight changes or partial actions of PUFAs on living cells.

In conclusion, we used distinct model membranes to clarify the specific effects of PUFAs on Ch-poor membranes. Future studies should identify the mechanism responsible for these effects, which will provide insight into the biological effects of PUFAs.

Acknowledgments The authors thank Nihon Suisan Kaisha, Ltd. for supplying DHA and EPA. The authors are very grateful to Ms. T. Tando for 
her kind assistance in the experimental work.

\section{References}

1) Carroll K. K., Am. J. Clin. Nutr., 53, 1064S-1067S (1991).

2) McLennan P., Howe P., Abeywardena M., Muggli R., Raederstorff D., Mano M., Rayner T., Head R., Eur. J. Pharmacol., 300, 83-89 (1996).

3) Dyerberg J., Bang H. O., Scand. J. Clin. Lab. Invest. 161 (Suppl.), 7 13 (1982)

4) Leaf A., Weber P. C., N. Engl. J. Med., 318, 549-557 (1988).

5) Menkes J. H., Alter M., Steigleder G. K., Weakley D. R., Sung J. H., Pediatrics, 29, 764-779 (1962).

6) Onuki Y., Morishita M., Takayama K., Tokiwa S., Chiba Y., Isowa K., Nagai T., Int. J. Pharm., 198, 147-156 (2000).

7) Morishita M., Kajita M., Suzuki A., Takayama K., Chiba Y., Tokiwa S., Nagai T., Int. J. Pharm., 201, 175-185 (2000).

8) Suzuki A., Morishita M., Kajita M., Takayama K., Isowa K., Chiba Y., Tokiwa S., Nagai T., J. Pharm. Sci., 87, 1196-1202 (1998).

9) Kajita M., Morishita M., Takayama K., Chiba Y., Tokiwa S., Nagai T. J. Pharm. Sci., 89, 1243-1252 (2000).

10) Onuki Y., Morishita M., Chiba Y., Tokiwa S., Takayama K., Chem Pharm. Bull., 54, 68-71 (2006).

11) Shaikh S. R., Cherezov V., Caffrey M., Stillwell W., Wassall S. R., Biochemistry, 42, 12028-12037 (2003).

12) Shaikh S. R., Brzustowicz M. R., Gustafson N., Stillwell W., Wassall S. R., Biochemistry, 41, 10593-10602 (2002).

13) Shaikh S. R., Dumaual A. C., Castillo A., LoCascio D., Siddiqui R. A., Stillwell W., Wassall S. R., Biophys. J., 87, 1752-1766 (2004).

14) Brown D. A., Rose J. K., Cell, 68, 533-544 (1992).

15) Simons K., Ikonen E., Nature (London), 387, 569-572 (1997).

16) Stulnig T. M., Huber J., Leitinger N., Imre E. M., Angelisova P., Nowotny P., Waldhausl W., J. Biol. Chem., 276, 37335-37340 (2001).

17) Matko J., Szollosi J., Immunol. Lett., 82, 3-15 (2002).

18) Stefanova I., Horejsi V., Ansotegui I. J., Knapp W., Stockinger H., Science, 254, 1016-1019 (1991)

19) Shenoy-Scaria A. M., Kwong J., Fujita T., Olszowy M. W., Shaw A. S., Lublin D. M., J. Immunol., 149, 3535-3541 (1992).

20) Orlowski S., Martin S., Escargueil A., Cell. Mol. Life Sci., 63, 1038 1059 (2006).

21) Lambert D., O’Neill C. A., Padfield P. J., Biochem. J., 387, 553-560
(2005).

22) Nusrat A., Parkos C. A., Verkade P., Foley C. S., Liang T. W., InnisWhitehouse W., Eastburn K. K., Madara J. L., J. Cell Sci., 113, 17711781 (2000).

23) London E., Biochim. Biophys. Acta, 1746, 203-220 (2005).

24) Veatch S. L., Keller S. L., Biophys. J., 85, 3074-3083 (2003).

25) Onuki Y., Morishita M., Takayama K., J. Controlled Release, 97, $91-$ 99 (2004).

26) Maitani Y., Nakamura K., Suenaga H., Kamata K., Takayama K., Nagai T., Int. J. Pharm., 200, 17-26 (2000).

27) Wang J., Megha London E., Biochemistry, 43, 1010-1018 (2004).

28) Brown D. A., London E., Annu. Rev. Cell Dev. Biol., 14, 111-136 (1998).

29) Ladbrooke B. D., Williams R. M., Chapman D., Biochim. Biophys. Acta, 150, 333-340 (1968).

30) Kahya N., Scherfeld D., Bacia K., Poolman B., Schwille P., J. Biol. Chem., 278, 28109-28115 (2003).

31) Dietrich C., Bagatolli L. A., Volovyk Z. N., Thompson N. L., Levi M., Jacobson K., Gratton E., Biophys. J., 80, 1417-1428 (2001).

32) Veatch S. L., Keller S. L., Biochim. Biophys. Acta, 1746, 172-185 (2005).

33) Heberle F. A., Buboltz J. T., Stringer D., Feigenson G. W., Biochim Biophys. Acta, 1746, 186-192 (2005).

34) Xu X., London E., Biochemistry, 39, 843-849 (2000).

35) Stillwell W., Wassall S. R., Chem. Phys. Lipids, 126, 1-27 (2003).

36) Shaikh S. R., Dumaual A. C., LoCassio D., Siddiqui R. A., Stillwell W., Biochem. Biophys. Res. Commun., 311, 793-796 (2003).

37) Duraisamy Y., Lambert D., O’Neill C. A., Padfield P. J., Biochem. Biophys. Res. Commun., 360, 885-890 (2007).

38) Li Q., Wang M., Tan L., Wang C., Ma J., Li N., Li Y., Xu G., Li J., J. Lipid Res., 46, 1904-1913 (2005).

39) Li Q., Tan L., Wang C., Li N., Li Y., Xu G., Li J., Eur. J. Nutr., 45 144-151 (2006).

40) Schley P. D., Brindley D. N., Field C. J., J. Nutr., 137, 548-553 (2007).

41) Fan Y. Y., McMurray D. N., Ly L. H., Chapkin R. S., J. Nutr., 133, 1913-1920 (2003).

42) Fan Y. Y., Ly L. H., Barhoumi R., McMurray D. N., Chapkin R. S., J. Immunol., 173, 6151-6160 (2004). 